\title{
The Superconducting Transition in Boron Doped Silicon Films
}

\author{
T. Kociniewsiki ${ }^{a, b}$, D. DéBArre ${ }^{a, b}$, A. Grockowiak ${ }^{c, d}$, J. KaČmarČíK ${ }^{c, e}$, C. Marcenat $^{c, *}$,

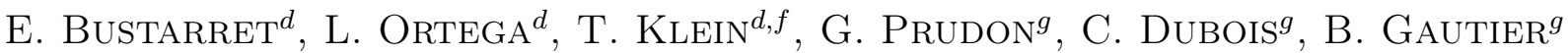 \\ AND J.C. DUPUY ${ }^{g}$ \\ ${ }^{a}$ Université Paris-Sud, Institut d'Electronique Fondamentale, UMR8622, 91405 Orsay, France \\ ${ }^{b}$ CNRS, 91405 Orsay, France \\ ${ }^{c}$ CEA-INAC/UJF-Grenoble 1, SPSMS, UMR-E 9001, 17 Rue des Martyrs, 38054 Grenoble, France \\ ${ }^{d}$ CNRS, Institut Néel, B.P. 166, 38042 Grenoble, France \\ ${ }^{e}$ Centre of Very Low Temperature Physics, IEP SAS and FS UPJŠ, 04001 Košice, Slovakia \\ ${ }^{f}$ Institut Universitaire de France and Université Joseph Fourier, B.P. 53, 38041 Grenoble, France \\ ${ }^{g}$ CNRS and INSA, Institut des Nanotechnologies de Lyon, 7 av. J. Capelle, 69621 Villeurbanne, France
}

We report on a detailed analysis of the superconducting properties of boron-doped silicon films grown along the 001 direction by gas immersion laser doping. This technique is proved to be a powerful technique to dope silicon in the alloying range $2-10$ at.\% where superconductivity occurs. The superconducting transitions are sharp and well defined both in resistivity and magnetic susceptibility. The variation of $T_{\mathrm{c}}$ on the boron concentration is in contradiction with a classical exponential dependence on superconducting parameters. Electrical measurements were performed in magnetic field on the sample with $c_{\mathrm{B}}=8$ at.\% (400 laser shots) which has the highest $T_{\mathrm{c}}(0.6 \mathrm{~K})$. No hysteresis was found for the transitions in magnetic field, which is characteristic of a type-II superconductor. The corresponding upper critical field was on the order of $1000 \mathrm{G}$ at low temperatures, much smaller than the value previously reported. The temperature dependence of $H_{\mathrm{c} 2}$ is very well reproduced by the linearized Gorkov equations neglecting spin effects in the very dirty limit. These measurements in magnetic field allow an estimation of the electronic mean-free path, the coherence length, and the London penetration depth within a simple two-band free electron model.

PACS numbers: 74.62.Dh, 73.61.Cw, 74.70.Ad

\section{Introduction}

Superconducting doped semiconductors have recently attracted a lot of interest (see [1] for a review). Among them silicon [2] is a special case as it is the technologically most important material and microelectronics processes could be applied to design new all-silicon hybrid (semiconducting-metallic-superconducting) devices.

To shed light on the interplay between disorder-induced localization, Coulomb interaction and superconductivity, we present here a systematic study of the evolution of the superconducting transition temperature as a function of boron doping in silicon.

\section{Experimental}

Thin films of boron doped silicon have been prepared by gas-immersion laser doping $[3,4]$. The surface of a silicon wafer is melted using laser pulses in the presence of a chemisorbed $\mathrm{BCl}_{3}$ gas. Boron diffuses into the liquid and is incorporated in the crystal upon cooling and recrystallization after the laser pulse (20-60 ns). This technique allows to exceed the solubility limit and to reach

* corresponding author; e-mail: christophe.marcenat@cea.fr a boron content in the range $2-10$ at.\%. The superconducting transition temperatures were determined from electrical resistivity measurements in a 4-contact geometry and from ac magnetic susceptibility experiments [5]. Quantitative determination of boron concentrations $c_{\mathrm{B}}$ were made by an isotopic comparative analysis of secondary ion mass spectroscopy (SIMS) profiles [6].

\section{Results}

Figure 1 represents the dependence of $T_{\mathrm{c}}$ on the boron content in a series of films with $c_{\mathrm{B}}$ ranging between 2 and 4 at.\%. Above 4 at.\% we observed previously [5] a non-intrinsic saturation of $T_{\mathrm{c}}$ probably related to a non-substitutional incorporation of boron. In the range of doping explored here, $T_{\mathrm{C}}$ is a quasi-linear function of $c_{\mathrm{B}}$ instead of the power law dependence, $T_{\mathrm{c}} \propto\left(c_{\mathrm{B}} / c_{\mathrm{C}}-1\right)^{0.5}$, $c_{\mathrm{C}}$ being a critical concentration, found previously not only in doped silicon [5] but also in doped diamond [7]. As a comparison the standard McMillan formula, $T_{\mathrm{c}} \propto$ $\exp \left(-1 /\left[\lambda_{\mathrm{e}-\mathrm{p}} /\left(1+\lambda_{\mathrm{e}-\mathrm{p}}\right)-\mu^{*}\right]\right)$ is reported in Fig. 1 with the Coulomb pseudopotential $\mu^{*}$ set equal to 0 (BCS limit) and 0.1 (typical value in metals). The electronphonon coupling constant $\lambda_{\mathrm{e}-\mathrm{p}}$ has been extrapolated linearly from virtual crystal approximation (VCA) calculations [8]. A free electron model with $\lambda_{\mathrm{e}-\mathrm{p}} \propto$ density of states $N\left(E_{\mathrm{F}}\right) \propto c_{\mathrm{B}}^{1 / 3}$ also fails to explain the data. 
The smooth decay of $T_{\mathrm{c}}$ cannot be reproduced by any exponential dependence on superconducting parameters.

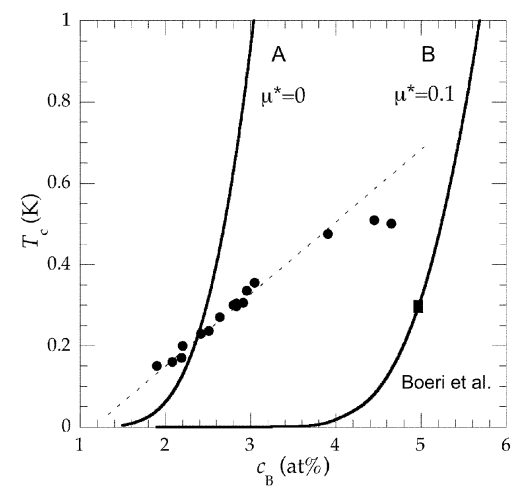

Fig. 1. Dependence of the superconducting transition temperature $T_{\mathrm{c}}$ on doping. The full lines are values obtained by the McMillan formula with the e-phonon coupling estimated by VCA approach, the Coulomb pseudopotential $\mu^{*}$ being equal to 0 (respectively 0.1 ) in line A (respectively B). The broken line is a linear fit for $2<c_{\mathrm{B}}<4$ at. $\%$.

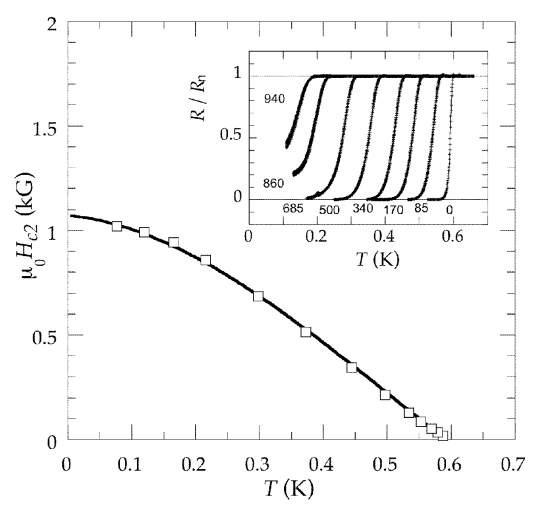

Fig. 2. Temperature dependence of the upper critical field. Full line is a fit by classical theory in the dirty limit. In the inset: temperature variation of the electrical resistance normalized to its normal state value in various magnetic fields given in G. The upper critical field was defined by the criterion where the ratio $R / R_{n}=0.9$.

In Fig. 2 we present the temperature dependence of the upper critical field $H_{\mathrm{c} 2}$ deduced from resistivity measurements at various fixed magnetic fields performed on the sample with $c_{\mathrm{B}}=8$ at.\% (400 laser shots) which has the highest $T_{\mathrm{c}}(0.6 \mathrm{~K})$. The transitions remained sharp as the magnetic field was increased and no hysteresis was observed which is consistent with a second-order transition and doped silicon being a type-II superconductor. The standard microscopic theory neglecting paramagnetic limitation reproduces the data very well (see full line in Fig. 2). Within a simple two-band free electron model one obtains an electronic mean free path $l \approx 2-3 \mathrm{~nm}$, a coherence length $\xi \approx 1000 \mathrm{~nm}$, and a London penetration depth $\lambda \approx 60 \mathrm{~nm}$. Comparing this $\lambda$ with the thickness, $80 \mathrm{~nm}$, of these films, we come to the conclusion that we might be in a quasi-2D regime.

\section{Conclusions}

Doped silicon should be intrinsically a type-I superconductor, $\kappa=\lambda / \xi \approx 1 / 15 \ll 1$, but is turned into a type-II system by strong impurity effects $[9]$.

The variation of $T_{\mathrm{c}}$ is in contradiction with a classical exponential dependence on superconducting parameters. Instead, $T_{\mathrm{c}}$ increases linearly with boron content raising the question of the importance of disorder and/or dimensionality.

\section{Acknowledgments}

This work was partly supported by French "Agence Nationale de la Recherche" (Projet SUSI BLAN08-3 317257), by Slovak Scientific Agency (VEGA 2/ $0148 / 10$ ), and by Slovak Research and Development Agency (contracts VVCE-0058-07, APVV-0346-07).

\section{References}

[1] X. Blase, E. Bustarret, C. Chapelier, T. Klein, C. Marcenat, Nature Mater. 8, 375 (2009).

[2] E. Bustarret, C. Marcenat, P. Achatz, J. Kačmarčík, F. Lévy, A. Huxley, L. Ortéga, E. Bourgeois, X. Blase, D. Débarre, J. Boulmer, Nature 444, 542 (2004).

[3] G. Kerrien, M. Hernandez, C. Laviron, T. Sarnet, D. Débarre, T. Noguchi, D. Zahorski, J. Venturini, M.N. Semeria, J. Boulmer, Appl. Surf. Sci. 208-209, 277 (2003).

[4] D. Cammilleri, F. Fossard, D. Débarre, C.T. Manh, C. Dubois, E. Bustarret, C. Marcenat, P. Achatz, D. Bouchier, J. Boulmer, Thin Solid Films 517, 75 (2008).

[5] C. Marcenat, J. Kačmarčík, R. Piquerel, P. Achatz, G. Prudon, C. Dubois, B. Gautier, J.C. Dupuy, E. Bustarret, L. Ortega, T. Klein, J. Boulmer, T. Kociniewski, D. Débarre, Phys. Rev. B 81, 020501 (2010).

[6] C. Dubois, G. Prudon, B. Gautier, J.C. Dupuy, Appl. Surf. Sci. 255, 1377 (2008).

[7] T. Klein, P. Achatz, J. Kačmarčík, C. Marcenat, F. Gustafsson, J. Marcus, E. Bustarret, J. Pernot, F. Omnes, E. Bo, C. Sernelius, A. Persson, Ferreira da Silva, C. Cytermann, Phys. Rev. B 75, 165313 (2007).

[8] L. Boeri, J. Kortus, O.K. Andersen, Phys. Rev. Lett. 93, 237002 (2007)

[9] A.L. Fetter, P.C. Hohenberg, in: Superconductivity Vol. 2, Ed. R.D. Parks, M. Dekker, New-York 1969, p. 187. 